\title{
ANÁLISE E INTERPRETAÇÃO DE DADOS GRAVIMÉTRICOS NAS PORÇÕES TERRESTRE E MARÍTIMA DO BRASIL MERIDIONAL
}

\author{
Paula Lucia Ferrucio da Rocha ${ }^{1,2}$ \& Luiz Fernando Santana Braga ${ }^{3}$ \\ 1- UFRJ, 2- ON/CNPq, 3- PETROBRÁS
}

\begin{abstract}
We have interpreted the Bouguer anomaly map from South Brazil and its adjoining oceanic areas, using the land data from the SAGP Project (1990), and the marine data derived from GEOSAT. With the aid of the vertical derivatives and the maximum horizontal gradientes of the Bouguer anomalies we have mapped the boundaries between the major lithospheric compartments. here characterized by their gravity signatures. Two dimensional gravity modelling with available geological constraints have also been performed aiming to cstimate the crustal thicknesses within each individual compartment. We show that the gravity responses of the continental, transitional and oceanic lithospheres are well distinguished.
\end{abstract}

\section{1- Introdução: Geologia e Mapeamento Geofísico}

Ao longo do século XX tem crescido a participação da Geofisica no estudo da Terra. Métodos fisicos são utilizados no estudo da atmosfera, dos oceanos, dos rios, dos ventos, do interior da Terra, na ecologia, e também no estudo dos minerais e das rochas da crosta terrestre.

Mais recentemente, métodos inicialmente criados para o estudo fisico da Terra, estão sendo utilizados no estudo de planetas, na chamada geologia comparada de planetas. Esse fato acentua a ligação da Geofisica com a Astronomia.

A gravimetria constitue um método tradicional de estudo fisico da Terra . A análise dos dados gravimétricos permite, por exemplo, a localização de estruturas geológicas em subsuperficie, o cálculo da profundidade de bacias sedimentares, o estudo isostático de uma área etc. Mapas gravimétricos Bouguer, ou alguns de seus produtos obtidos através de filtragens (mapa residual, mapa regional, mapa de gradiente vertical, etc.) usualmente apresentam 
anomalias, ou provincias, geofisicas fortemente correlacionáveis às estruturas geológicas mapeadas em superficie.

Pode-se, portanto, a partir das medidas do campo gravitacional da Terra, delimitar, em mapa, provincias que apresentam diferentes contrastes gravimétricos que refletem diferenças laterais de densidades entre os materiais em subsuperficie. Conhecendo-se a gênese dos diversos sitios geológicos mapeáveis em superficie, esta nova informação pode definir espessuras verticais e limites laterais de corpos em subsuperficie que afloram de maneira muito timida sem definição para um bom mapeamento geológico.

As medidas do campo magnético da Terra, por exemplo, são usadas para definir em mapa anomalias magnéticas que podem corresponder a diversas feições geológicas, como por exemplo um dique de basalto ou lineamentos de diques, como mostra o Mapa do Campo Magnético Anômalo do Brasil (Moraes e Braga, 1993) na região do Arco de Ponta Grossa na Bacia do Paraná, ou ainda, em escala regional, limites de bacias sedimentares. Estas anomalias são produzidas pelo contraste no magnetismo das rochas.

Cabe lembrar que as rochas são constituidas por minerais que apresentam comportamentos diferentes quando estão sob a influência de um campo magnético. Os minerais são classificados, de acordo com suas propriedades magnéticas, como ferrimagnéticos, ferromagnéticos, anti-ferromagnéticos, diamagnéticos e paramagnéticos.

\section{2- Objetivos do trabalho}

Segundo Brito Neves (1995), "um continente é uma acumulação grande e isostaticamente positiva da litosfera/crosta continental, soma algébrica positiva de processos de acresção, fragmentação (fissão) e re-aglutinação (fusão). Uma vez circunstancialmente reunidos, os tipos crustais passam a agir com caracteristicas tectônicas de conjunto (por exemplo, América do Sul), mas os seus tipos crustais conseguem preservar peculiaridades geológicas e geofisicas próprias e intransferiveis".

Neste estudo procuramos identificar, a partir do campo anômalo de gravidade, peculiaridades geofisicas dos diversos tipos crustais que compõem a litosfera das regiões sul e sudeste do Brasil. Embora os dados geofisicos utilizados neste trabalho estejam em escala regional, os resultados obtidos nos permitiram, além de chegar a uma nova visão de 
conjunto da subsuperficie do Brasil na área em estudo, alcançar certos resultados locais, referentes à espessura crustal, que iluminam a história geológica do continente.

No Brasil a cobertura gravimétrica ainda é relativamente pequena, com exceção de áreas sedimentares produtoras de petróleo. Apesar disto, a densidade de infomações gravimétricas existentes na região sul e sudeste do Brasil e nas áreas oceânicas adjacentes se prestam a determinadas análises, em escala regional, que abrem a possibilidade de uma modelagem dos blocos tectônicos, de grande importância para o conhecimento da geologia da área. Neste trabalho identificamos os limites de grandes blocos com mesma identidade gravimétrica, ou seja, os limites de blocos tectônicos. O Mapa Geológico do Brasil (Schobbenhaus et al., 1981), escala 1:2.500.000 e o Mapa Tectono-Geológico do Brasil (Delgado et al., 1996), escala 1:7.000.000, deram o suporte com a geologia de superficie para a interpretação dos dados geofisicos.

\section{3- $\mathrm{A}$ área de trabalho}

A área estudada, figura 1 , é limitada pelos paralelos de $16^{\circ} \mathrm{S}$ e $33^{\circ} \mathrm{S}$ e pelos meridianos $35^{\circ} \mathrm{W}$ e $58^{\circ} \mathrm{W}$, compreendendo, a parte terrestre, os estados da região sul (Rio Grande do Sul, Santa Catarina,Paraná), da região sudeste (São Paulo, Rio de Janeiro, Espírito Santo e boa parte de Minas Gerais) e os da região centro-oeste (Mato Grosso do Sul e sul de Goias). $\mathrm{Na}$ parte marítima, estão as bacias marginais de Campos, Santos e Pelotas e a região marinha adjacente.

Com os processos que sofreu ao longo do tempo geológico, a litosfera do Brasil apresenta hoje uma geologia muito complexa. Temos bacias sedimentares intracratônicas bordejadas por faixas de embasamento pré-cambriano aflorante e bacias marginais ao longo de toda a costa do Brasil representando um papel fundamental para a economia do pais na produção de petróleo e gás.

Conforme já foi dito antes, face à falta de resolução dos nossos dados, buscamos como apoio deste trabalho uma geologia regional (descrita sem muitos detalhes), no Mapa Geológico do Brasil (Schobbenhaus et al., 1981), escala 1:2.500.000 e no Mapa TectonoGeológico do Brasil (Delgado et al., 1996), escala 1:7.000.000. 
A área em estudo é subdividada geologicamente, conforme mostra a figura 2 (adaptado do Mapa Tectono-Geológico do Brasil (Delgado et al., 1996), escala 1:7.000.000), nos seguintes compartimentos tectono-geológicos: Os Cinturões Neoproterozóicos, Meso a Neoproterozóicos e Paleoproterozóicos; a Bacia Sedimentar do Paraná; e os núcleos e fragmentos Cratônicos Arqueanos. Na área costeira, temos as bacias marginais de Campos, Santos e Pelotas.

\section{4- Dados Gravimétricos}

Os dados gravimétricos, originalmente adquiridos em forma de grid digital com $5 \mathrm{~km} \mathrm{X}$ $5 \mathrm{~km}$, foram obtidos em banco de dados diferentes: SAGP (1990) para dados terrestres; GEOSAT para dados maritimos.

a) Dados Terrestres - O Projeto SAGP (1990) (South American Gravity Project), foi executado pelo Departamento de Cièncias da Terra da Universidade de Leeds em colaboração com o Prof. Watts, do Departamento de Ciências da Terra da Universidade de Oxford, sob o patrocinio do Serviço Industrial da Universidade de Leeds, Ltda. O projeto foi patrocinado por várias companhias, entre as quais, Agip, Amoco, Arco, BP, Conoco, Exxon, Marathon, Mobil, Petrobrás, Shell, Texaco and Unocal; também participaram a YPF (Argentina) e a COPI.

Os dados gravimétricos observados ( $\mathrm{g}_{\mathrm{obs}}$ ) foram ajustados para o datum IGSN71. Essa datum foi usado como referência para a rede sul-americana de estações base "Sistema Informativo Latinoamericano de Gravedad" (SILAG77) criado pelo Instituto Panamericano de Geografia e Historia (IPGH) em colaboração com o Servicio Geodésico Inter Americano. Também foi usada a "Rede Gravimétrica Fundamental Brasileira" (Departamento de Geofisica, $\mathrm{MCT} / \mathrm{CNPq}$, Observatório Nacional)

Para o cálculo da gravidade normal ( $\gamma_{84}$, ou gravidade teórica , ou correção de latitude), o Projeto SAGP utilizou o World Geodetic System 1984 (WGS84) para definir a fórmula de gravidade normal, numericamente dada por:

$$
\gamma_{84}=978032.67714\left(\frac{1+0.00193185138639 \sin ^{2} \varphi}{\sqrt{\left(1-0.00669437999013 \sin ^{2} \varphi\right)}}\right) \mathrm{m} \mathrm{Gal}
$$

onde $\varphi$ é a latitude 
A principal diferença entre o WGS84 e o Geodetic Reference System,1980 GRS80 é a inclusão no WGS84 de toda a massa da atmosfera terrestre. Ao aplicar o WGS84, um termo adicional, $\delta \mathrm{g}_{\mathrm{A}}$, deve ser somado à expressão da anomalia.

Anomalia de gravidade $=\mathrm{g}_{0 \mathrm{bx}}-\gamma_{84}+\delta \mathrm{g}_{\mathrm{A}}+$ termos de redução de gravidade

onde $\delta g_{A}$ é a correção de gravidade atmosférica, visto que $\gamma_{84}$ é determinado a partir do nivel médio do mar. A equação empirica usada para calcular $\delta g_{A}$ é:

$$
\delta g_{\Lambda}=0,87 e^{0.116 h} \mathrm{mGal}
$$

onde $\mathrm{h}=\mathrm{H}^{1,047} \quad(\mathrm{H}$ é a elevação em quilômetros). Para $\mathrm{H}<0$, faz-se $\mathrm{H}=0$.

Para a Correção de "Free Air", foi utilizado o valor de $0,3086 \mathrm{mGal} / \mathrm{metro}$. Além disso, foi introduzido uma correção de latitude e altura, segundo Balmino (1990) in SAGP (1990).

Para a Correção de Bouguer e redução da densidade, utilizou-se a fórmula da placa infinita com densidade $\rho\left(\mathrm{g} / \mathrm{cm}^{3}\right)$ e espessura h (metros).

Correção de Bouguer $=0,04191 \rho \mathrm{h} \mathrm{mGal}$ $\operatorname{com} \rho=2,67 \mathrm{~g} / \mathrm{cm}^{3}$ na parte terrestre e $\rho=2,2 \mathrm{~g} / \mathrm{cm}^{3}$ na parte maritima.

Nas Correções de Terreno $(\mathrm{T} \rho)$ e de Curvatura da Terra , utilizou-se a fórmula de massa em linha (line mass formula) (Kane (1962) in SAGP (1990)) para calcular a Correção de Terreno no entorno de cada ponto do grid até 166,7 km com os efeitos dos 4 pontos mais internos do grid ignorados e levando-se em conta a curvatura da Terra.

Para a Correção de Curvatura da Terra, foi usada a expressão dada por Cordell et al. (1982) in SAGP 1990). 
Correção de Curvatura $=-1,4639108 \times 10^{-3} \mathrm{~h}+3,532715 \times 10^{-7} \mathrm{~h}^{2}-4,449648 \times 10^{-14} \mathrm{~h}^{3}$ $\mathrm{mGal}$

onde $\mathrm{h}$ é a altitude do ponto observado em metros.

O projeto SAGP, (1990) considera que, além das Correções de Terreno, os erros cometidos na localização e na cota das estações gravimétricas são o fator de maior peso na estimativa das correções gravimétricas. $\mathrm{O}$ erro na anomalia de Bouguer $(\Delta \mathrm{g})$ é relacionado ao erro na determinacão da latitude pela seguinte fórmula:

$$
\Delta \mathrm{g}=0,81 \sin 2 \varphi \mathrm{mGal} / \mathrm{km},
$$

onde $\varphi$ é latitude geodésica, isto é, um erro de $1 \mathrm{~km}$ na localização pode chegar a introduzir um erro máximo de $0.81 \mathrm{mGal}$ no cálculo da anomalia de Bouguer.

Em condições atmosféricas normais, as medidas de altitude (cota) pelo método barométrico apresentam uma incerteza entre $\pm 2 \mathrm{~m}$ e $\pm 5 \mathrm{~m}$. Isso é equivalente a um erro de $\pm 0,4 \mathrm{mGal} a \pm 1,0 \mathrm{mGal}$ no valor da Anomalia Bouguer

A Anomalia de "Free Air", a Anomalia de Bouguer Simples e a Anomalia de Bouguer Completa são definidas como:

-Anomalia de "Free Air" (AFA) $=\mathrm{g}_{\mathrm{obs}}-\gamma_{84}+\delta \mathrm{g}_{\mathrm{A}}+0,3086 \mathrm{~h}$

-Anomalia de Bouguer Simples $=($ AFA $)-0,04191 \mathrm{ph}$

-Anomalia de Bouguer Completa $=($ AFA $)-0,04191 \rho h+T \rho+$ Correção de Curvatura

b) Dados Marítimos - Foram gerados a partir de altimetria de satélite Geosat, disponiveis na Internet, em 1995. O Geosat (satélite geodésico da marinha americana) foi lançado em 12 de março de 1985 realizando 2 missões: na primeira, com fins geodésicos (GM - Geodetic Mission, 1985-1986), coletou dados de altitude do mar entre os paralelos de $72^{\circ} \mathrm{N}$ e $72^{\circ} \mathrm{S}$ ao longo de trajetórias com tracks de aproximadamente $3 \mathrm{~km}$ de espaçamento na latitude de $30^{\circ} \mathrm{S}$ resultando em uma resolução de $15 \mathrm{~km}$. Na segunda, com objetivos ocenográficos 
(ERM - Exact Repeat Mission, 1986-1990), coletou dados muito espaçados (aproximadamente $140 \mathrm{~km}$ na latitude de $30^{\circ} \mathrm{S}$, sendo os dados gravimétricos limitados à resolução para comprimentos de onda de aproximadamente 260 km, Aguiar, 1997.

\section{5- Mapas Gravimétricos}

O Mapa Bouguer, figura 3, foi construido a partir do grid original do Projeto SAGP (1990) e confeccionado com o método de gridagem de minima curvatura do pacote de "software" GEOSOFT Inc., (1994) com espaçamento de 5km, todos na escala 1:2.500.000, coordenada policônica e meridiano central de $54^{\circ}$, na mesma janela mostrada na figura 1.1.

O Mapa de Lineamentos Gravimétricos, figura 3, obtido com o auxilio do Mapa de Gradientes Verticais Máximos - construido com o pacote de programas Boundary (USGS, 1992) . O Mapa Geológico do Brasil escala 1:2.500.000 (Schobbenhaus et al, 1981 ) serviu como suporte para as interpretações dos lineamentos gravimétricos, ou seja, ofereceu informações sobre limites das bacias, faixas dobradas, lineamentos geológicos regionais etc.

\section{6- Compartimentação Gravimétrica}

As anomalias gravimétricas Bouguer, mapeadas sobre as áreas de embasamento aflorantes, se correlacionam fortemente com as estruturas apresentadas no Mapa Geológico do Brasil (Schobbenhaus et al., 1981), escala 1:2.500.000.

No mapa Bouguer da área, figura 3, destacam-se três grandes compartimentos litosféricos: a litosfera continental (LC) com espessuras crustais normais e afinamentos restritos aos depocentros da Bacia do Paraná; a litosfera transicional (LT) ao largo da margem continental, onde a crosta continental regionalmente se afina em direção a águas profundas; e a litosfera oceânica (LO), sob águas maritimas com profundidades que ultrapassam a cota de 3500 metros.

A LC, na região sudeste do Brasil, se caracteriza por valores de gravidade Bouguer relativamente baixos, onde dominam as isogálicas na faixa $-120 \mathrm{a}-70 \mathrm{mGal}$. Internos à LC, são bem identificados gravimetricamente: as fronteiras da Faixa Brasília com o Cráton de São Francisco e o Bloco Paranapanema subjacente à Bacia do Paraná; o limite oeste do Bloco 
Paranapanema com a Faixa Paraguai; e a faixa costeira do cinturão de gnaisses do Arqueano sobre o qual as anomalias Bouguer maiores que $-70 \mathrm{mGal}$ indicam sua porção onshore distendida durante o rifteamento do Gondwana.

A LT apresenta um gradiente regional Bouguer com isogálicas que se elevam de aproximadamente $-20 \mathrm{mGal}$, ao longo da linha de costa, para valores da ordem de $120 \mathrm{mGals}$ em águas profundas. Sobrepostas à LT, dentro dos limites da área estudada, se encontram as Bacias do Espirito Santo, Campos e Santos; seus limites Leste e Oeste são bem marcados por fortes lineamentos no mapa do campo gravimétrico Bouguer. Tais lineamentos a Oeste se associam às zonas de charneira e a leste registram a mudança de crosta continental para crosta oceânica.

A LO apresenta valores Bouguer na faixa de 120 a $220 \mathrm{mGal}$, crescentes em direção à Cordilheira Meso-Atlântica onde a espessura da crosta oceânica atinge valores minimos.

\section{7-O perfil gravimétrico}

Para a determinação da espessura crustal ao longo do perfil gravimétrico A-A' , indicado na figura 3, foi feita uma modelagem 2D das estruturas em subsuperficie, utilizando informą̧ões regionais de densidade e geometria dos principais compartimentos geológicos em superficie. Determinou-se a forma e a espessura crustal dos três compartimentos litosféricos: Crosta da LC com densidade de 2,78 - (Bacia do Paraná, a Faixa Brasilia, Cráton de São Francisco e a faixa costeira). Crosta da LT com densidade de 2,78 e Crosta da LO com densidade de 2,80 .

A modelagem foi executado com o programa GM-SYS do pacote GEOSOFT Inc. (1994). Este algoritmo, que resolve o problema em duas dimensões, é utilizado do seguinte modo: inicialmente, dado um contraste de densidade, simula-se uma distribuição geométrica de massa, em subsuperficie. $\mathrm{O}$ campo resultante dessa distribuição hipotética é comparado com o campo realmente observado no perfil gravimétrico. Este procedimento é repetido, até que se chegue a um resultado satisfatório, isto é, até que o valor teórico calculado na simulação corresponda ao campo observado. 


\section{8- Análise dos Resultados}

Entre os resultados importantes obtidos neste estudo destacamos o Mapa de Lineamentos Gravimétricos apresentado na figura $3 \mathrm{e}$ a modelagem dos blocos tectônicos em subsuperficie, apresentada na figura 4. Os valores encontrados para espessura crustal da LC, obtidos na modelagem do perfil gravimétrico, são típicos, isto é atingem $32-34 \mathrm{~km}$ sob a Bacia do Paraná, chegando a $38-40 \mathrm{~km}$ nas áreas onde o embasamento aflora. Usamos para modelo da distribuição sedimentar as isópacas de sedimentos da bacia do Paraná (Zalán et al., 1990).

Na LT, a crosta continental se afina em direção ao oceano e, possivelmente, é penetrada por materiais básicos (diques e sills) de origem mantélica (Brito Neves, 1995) . Nela, os valores da espessura crustal se reduzem gradativamente de $30-35 \mathrm{~km}$ sob a faixa costeira até valores de 6-8 km sob lâminas d'água da ordem de 3000-3500 metros.

$\mathrm{Na} \mathrm{LO}$, as espessuras crustais oscilam entre valores na faixa de 5 a $7 \mathrm{~km}$.

No Mapa Bouguer, apresentado na figura 3, se observam valores positivos e negativos de gravidade anômala. De um modo geral, as anomalias muito negativas estão associadas a montanhas e áreas de alta elevação na superficie da Terra, indicando uma raiz de compensação, e os valores muito positivos se encontram nas regiões oceânicas, indicando um manto mais próximo à superficie devido ao afinamento crustal.

\section{Bibliografia:}

Aguiar,A.C.K.V., 1997 . Estudo Gravimétrico da Morfologia e da Estrutura Crustal 3D da Porção Ocidental do Atlântico - Sul entre os Paralelos $18^{\circ} \mathrm{S}$ e $40{ }^{\circ} \mathrm{S}$. Tese de Mestrado- ON, Fevereiro.

Balmino, G., 1990 , pers comm., (BGI, Toulouse, France), in SAGP ,1990.

Brito Neves, B.B., 1995 . Boletin IG-USP, Série Didática no. 7, Universidade de São Paulo IG-USP.

GEOSAT, 1995 , (satélite geodésico da marinha americana) - banco de dados disponivel na internete

Kane, M.F., 1962, A comprehensive system of terrain corrections using a digital computer. Geophysics, 27, 455-462, in SAGP , 1990 
GEOSOFT Inc.,1994, Mapping and Processing System , February, 1994 - Manual de Instruções

Delgado et al., 1996. Mapa Tectono-Geológico do Brasil escala 1:7.000.000, DNPM. Moraes, R.A.V. e Braga,L.F.S., 1993. Mapa do Campo Magnético Anômalo do Brasil e da Área Oceânica Adjacente, Anais do $3^{\circ}$ Congresso Internacional da Soc. Brasileira de Geofisica - Rio de Janeiro, p. 826-829.

SAGP ,1990. South American Gravity Project. Progress Report Number 2 , December

Schobbenhaus et al., 1981. Mapa Geológico do Brasil escala 1:2.500.000, DNPM.

Cordell , L., Keller, G.R. and hildenbrand, T.G., 1982 , Gouguer gravity map of the Rio Grande Rift, Colorado, New Mexico and Texas. Map GP-949 USGS 1:1.000.000 series, in SAGP, 1990.

USGS, 1992 United States Department of the Interior Geological Survey, - Open-File Report.

Zalán, P.V., Wolf, S., Astolfi,M.A.M., Vieira, I.S., Conceição, J.C.J., Appi, V.T., Neto, E.V.S., Cerqueira, J.R., Marques, A., 1990. Interior Cratonic Basins, chapter 33, edited by M.W.Leighton, D.R.Kolata, D.F. Oltz, and J.J.Eidel: American Association of Petroleum Geolocists Menoir 51, 819p. 


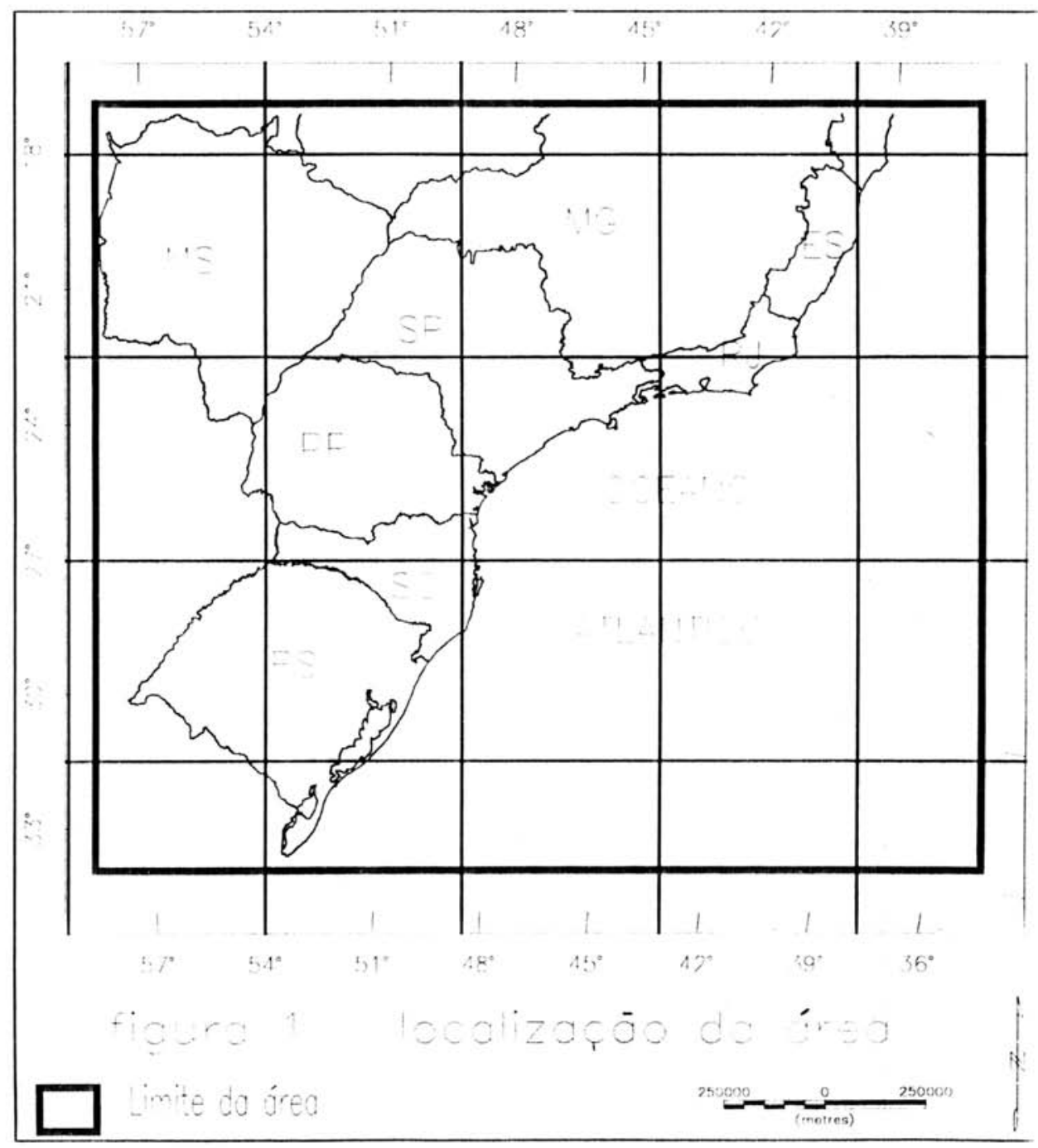




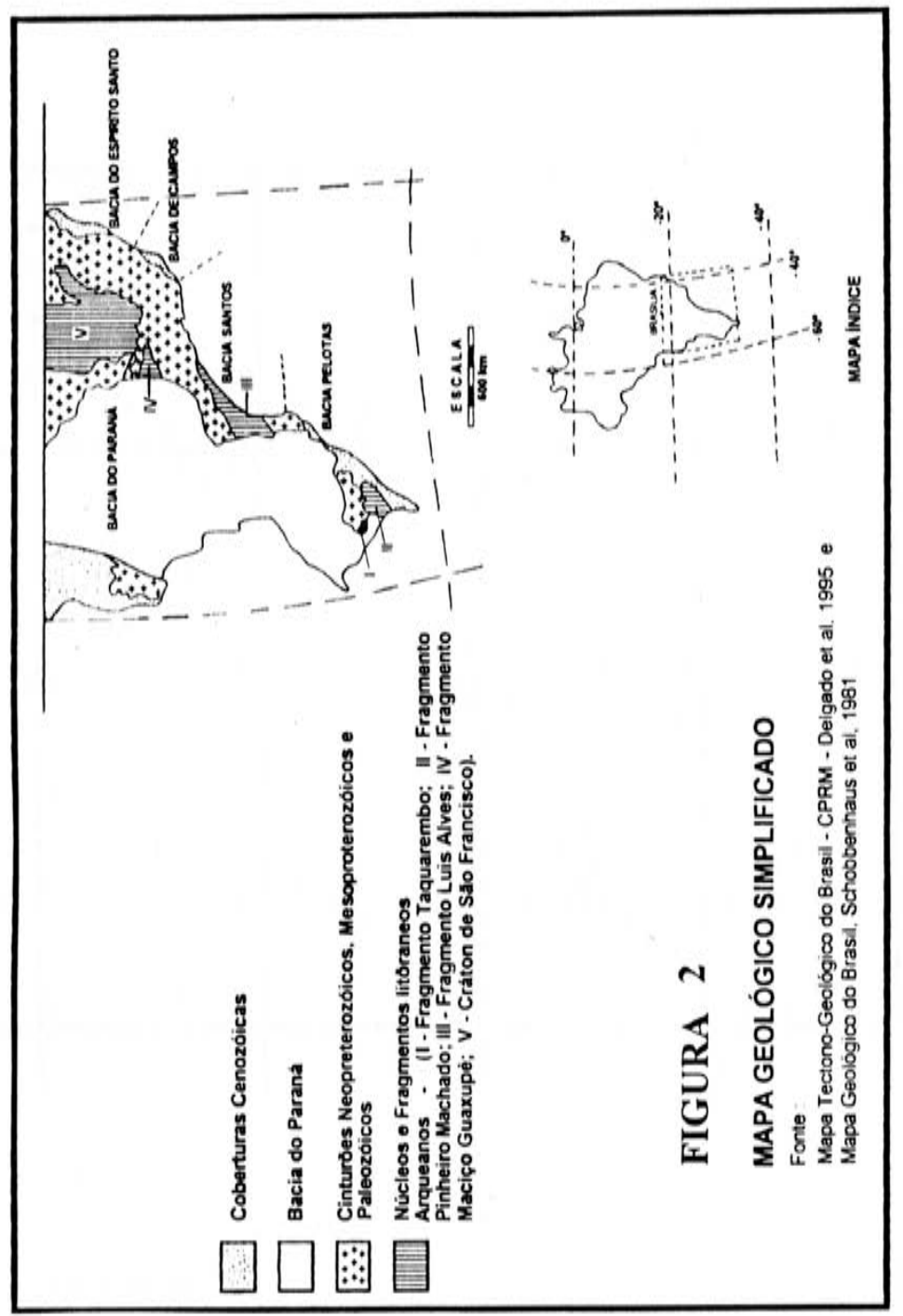




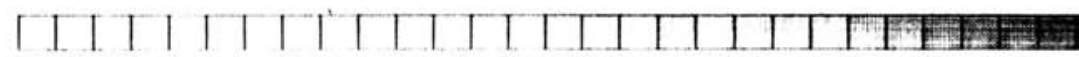

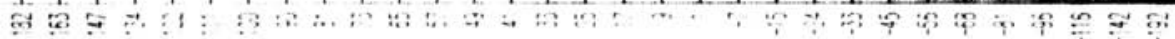

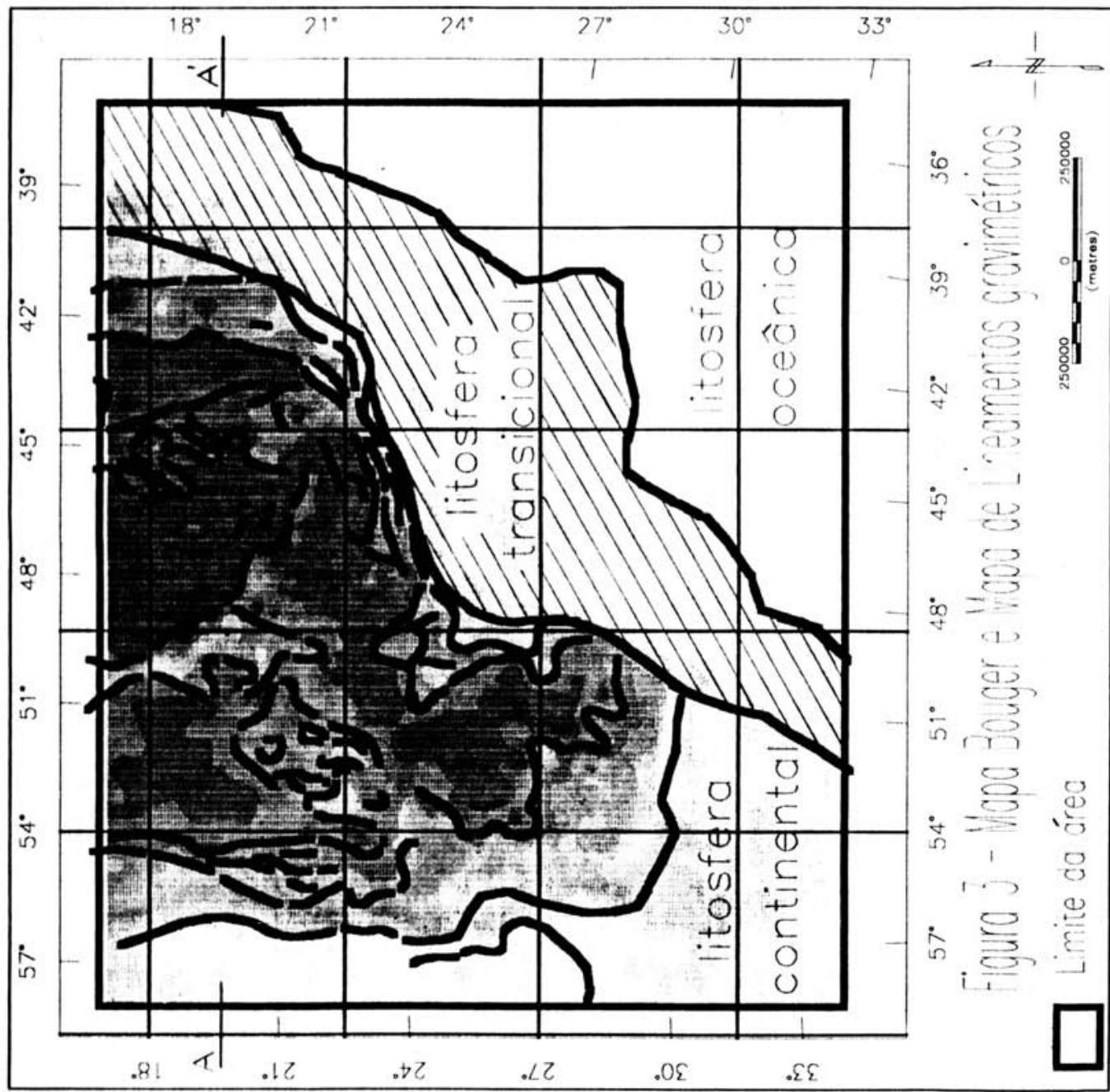



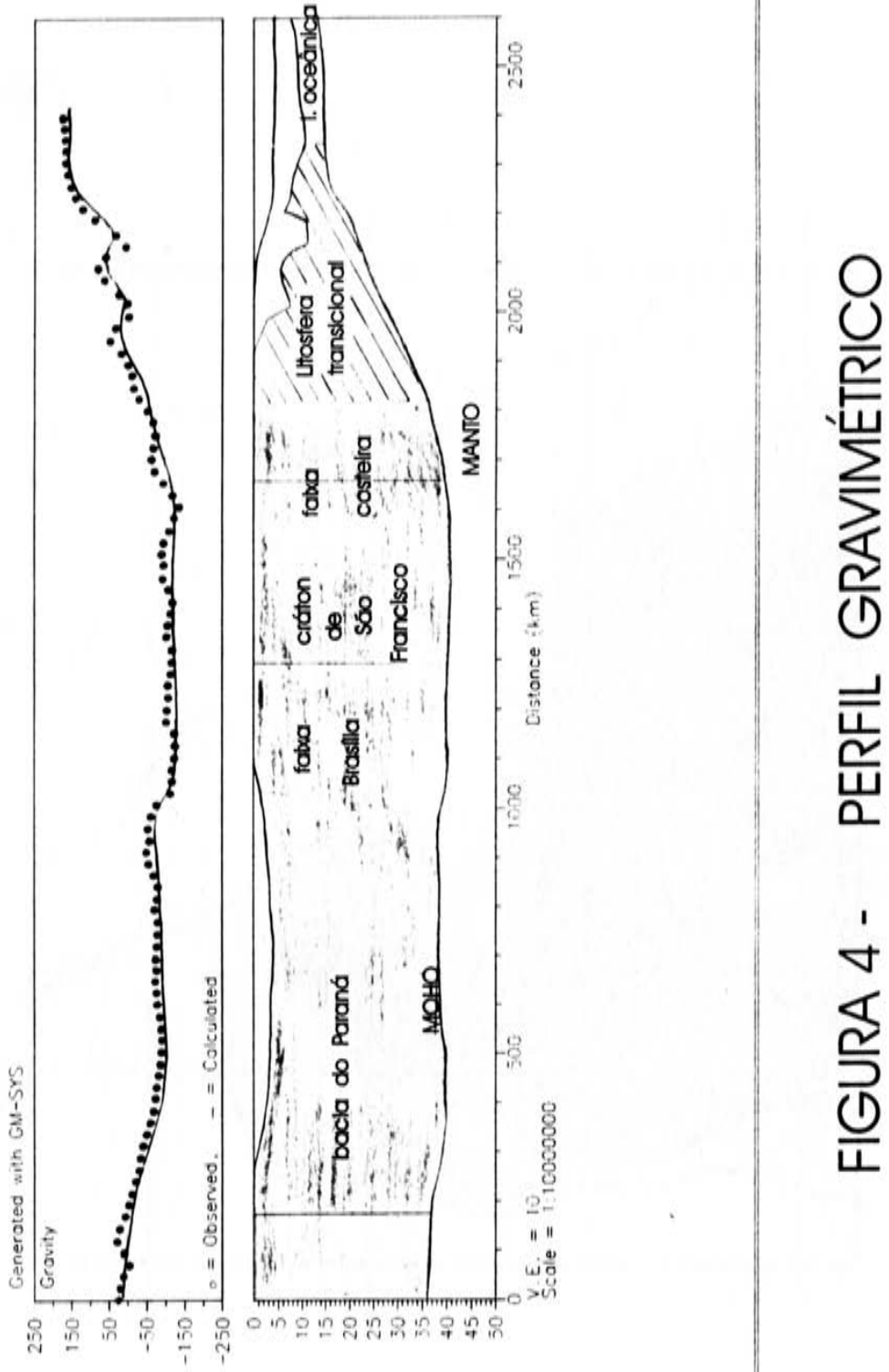